\title{
Magnetic field amplification by high Alfven Mach number shocks in partially ionized plasmas
}

\author{
Yutaka Ohira* \\ Aoyama Gakuin University \\ E-mail: ohira@phys.aoyama.ac.jp
}

\begin{abstract}
The interstellar medium and ejecta of supernova are not always completely ionized. Such partially ionized plasmas are though to be unsuitable for cosmic ray acceleration. In order to study shock structures of collisionless shocks in partially ionized plasmas, we perform two-dimensional hybrid simulations. We find that large density fluctuations and large magnetic fields fluctuations are generated both in the upstream and downstream regions for high Alfvén Mach number shocks. For the shock velocity $v_{\mathrm{s}}=1333 \mathrm{~km} / \mathrm{s}=57 v_{\mathrm{A}}$, the strong turbulence is generated in the downstream region by the upstream density fluctuations and the downstream magnetic field is amplified over 30 times the initial upstream magnetic field. Observed shock structures suggest that diffusive shock acceleration can operate at perpendicular shocks propagating into partially ionized plasmas in real three-dimensional systems.
\end{abstract}

The 34th International Cosmic Ray Conference

30 July- 6 August, 2015

The Hague, The Netherlands

* Speaker. 


\section{Introduction}

Cosmic rays (CRs) are thought to be accelerated by collisionless shocks driven by supernova explosions. Gamma-ray observations of supernova remnants (SNRs) show that SNRs accelerate CRs [1]. The most plausible acceleration mechanism is diffusive shock acceleration (DSA) [2, 3]. In order to accelerate CRs to the knee energy $\left(10^{15.5} \mathrm{eV}\right)$, magnetic fields around SNR shocks must be amplified over $100 \mu \mathrm{G}$. In fact, several observations suggest that magnetic fields are amplified over $100 \mu \mathrm{G}$ around SNR shocks [4, 5, 6, 7]. There are many studies about the magnetic field amplification around the SNR shock, but it is still an open problem.

The interstellar medium and ejecta of SNRs are not always fully ionized plasmas. Plasma instabilities are often thought to be stabilized in the partially ionized plasma because of collisions with neutral particles. However, it was recently proposed that many plasma instabilities are excited by ions produced by ionization around a collisionless shock [8, 9, 10, 11].

By performing hybrid simulations of a collisionless shock wave propagating into a partially ionized plasma, Ohira (2013) [12] showed that ionization of neutral particles excites plasma instabilities both in the upstream and downstream regions. In addition, the simulation showed that some downstream hot hydrogen atoms leak into the upstream region and density fluctuations are produced by an acoustic instability due to the leaking hydrogen atoms [11]. The shock velocity of the first hybrid simulation of Ohira (2013) is $v_{\mathrm{sh}} \approx 2700 \mathrm{~km} / \mathrm{s}$. For a slower shock velocity, more hydrogen atoms are expected to leak into the upstream region $[13,14]$ and the amplitude of the upstream density fluctuation is expected to be larger. Therefore, we would naively expect that magnetic fields could be amplified by the upstream density fluctuations if the shock velocity is smaller and the Alfvén Mach number is larger than that of Ohira (2013). In fact, magnetohydrodynamics simulations showed that upstream density fluctuations amplify magnetic fields in the downstream region if the Alfvén Mach number and amplitude of the density fluctuations are sufficiently large $[15,16]$. Therefore, in this study, we perform two-dimensional hybrid simulations of collisionless shocks propagating into the partially ionized plasmas for $v_{\mathrm{sh}} \approx 57 v_{\mathrm{A} \text {,tot }} \approx 1333 \mathrm{~km} / \mathrm{s}$, that are larger Alfvén Mach number and slower shock velocity than that of Ohira (2013).

\section{Simulation setup}

To study collisionless shocks propagating into partially ionized plasmas, we use a two dimensional hybrid code. The code solves charge exchange and collisional ionization of hydrogen atoms [12]. The ionization timescale is at least $10^{5}$ times longer than the gyro period for typical young SNRs. In order to reduce the computational cost, we adopt cross sections boosted by a factor of $10^{3}$, but ionization timescale is still much longer than the gyro period.

Because hybrid codes do not solve the electron dynamics, we need an assumption for the electron temperature, $T_{\mathrm{e}}$. Hence, we assume that the relative velocity between a hydrogen atom and electrons is given by [17]

$$
v_{\mathrm{rel}, \mathrm{eH}}=\sqrt{\frac{8 k T_{\mathrm{e}}}{\pi m_{e}}+\left|\vec{v}_{\mathrm{H}}-\vec{u}_{\mathrm{p}}\right|^{2}}
$$

where $\vec{v}_{\mathrm{H}}$ is the velocity of the hydrogen atom and the mean velocity of electrons is assumed to be the mean velocity of protons, $\vec{u}_{\mathrm{p}}$. According to the early studies, the electron temperature is 

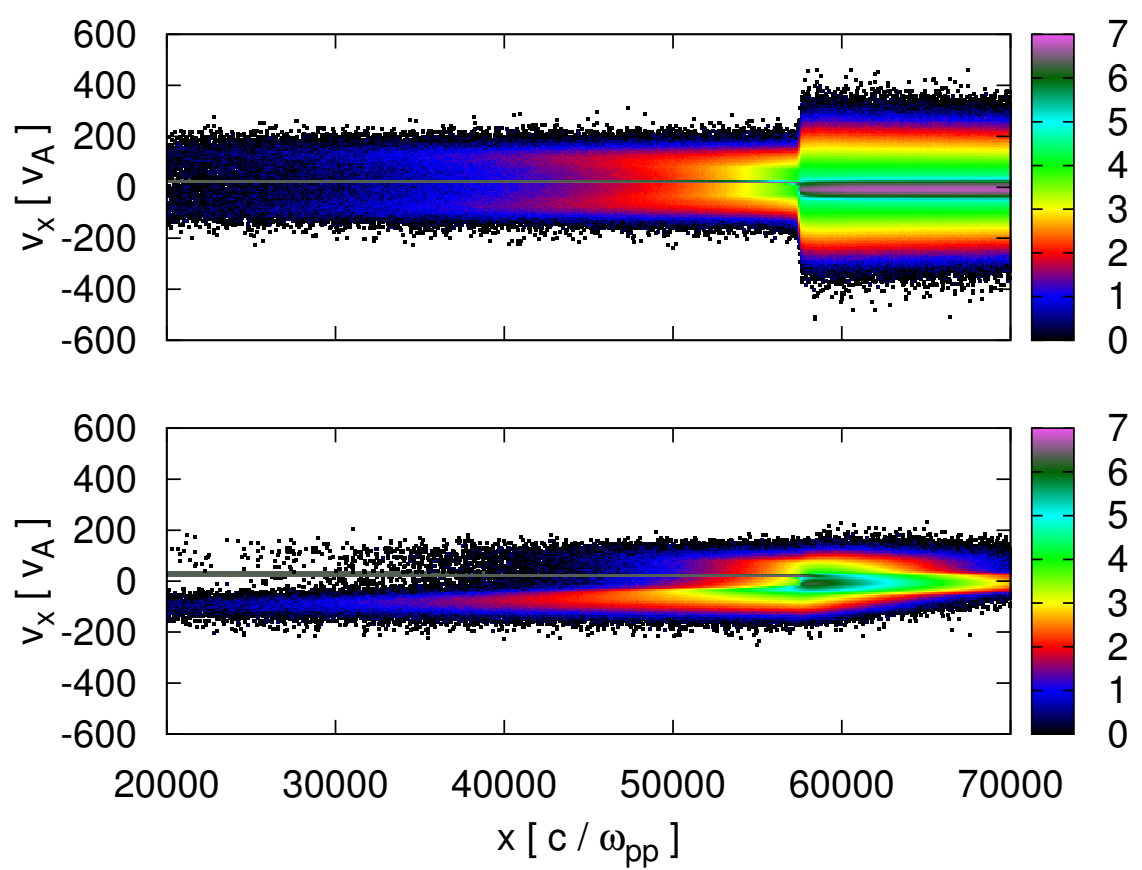

Figure 1: Phase space plots of protons (top) and hydrogen atoms (bottom) at $t=2000 \Omega_{\mathrm{cp}}^{-1}$. The color shows the phase space density in logarithmic scale.

expected to be $T_{\mathrm{e}} \approx 0.01 m_{\mathrm{p}} v_{\mathrm{d}}^{2} / 3$ in the downstream region of SNRs [18, 19, 20], where $v_{\mathrm{d}}$ is the upstream flow velocity in the downstream rest frame. Therefore, in this study, we assume $T_{\mathrm{e}}=0$ and $T_{\mathrm{e}}=0.01 m_{\mathrm{p}} v_{\mathrm{d}}^{2} / 3$ in the upstream and downstream regions, respectively.

We set a two-dimensional simulation box in the $x y$ plane with the periodic boundary condition in the $y$ direction. Simulation particles are injected at the left boundary, $x=0$, and reflect at the right boundary. Then, a shock propagates to the left boundary, that is, the simulation frame is the downstream rest frame. The size of the simulation box is $L_{\mathrm{x}} \times L_{\mathrm{y}}=77600 \mathrm{c} / \omega_{\mathrm{pp}} \times 800 \mathrm{c} / \omega_{\mathrm{pp}}$, where $c$ and $\omega_{\mathrm{pp}}$ are the speed of light and plasma frequency of protons, respectively. The cell size and time step are $\Delta x=\Delta y=c / \omega_{\mathrm{pp}}$ and $\Delta t=8.333 \times 10^{-3} \Omega_{\mathrm{cp}}^{-1}$, respectively. Initially, the magnetic field is taken to be spatially homogenous, pointing in the $y$ direction, $B=B_{0} e_{\mathrm{y}}$. Therefore, the shock in this simulation is a perpendicular shock, where the shock normal direction is perpendicular to upstream mean magnetic field lines.

Parameters of the upstream plasma are as follows. The upstream ionization fraction is $f_{\mathrm{i}}=$ 0.5 , the plasma beta is $\beta_{\mathrm{p}}=\beta_{\mathrm{H}}=0.5$, and the drift velocity of the $x$ direction is $v_{\mathrm{d}}=30 v_{\mathrm{A}}=$ $1000 \mathrm{~km} / \mathrm{s}$, where $v_{\mathrm{A}}=B_{0} / \sqrt{4 \pi \rho_{\mathrm{p}, 0}}$ is the Alfvén velocity defined by the proton mass density in the far upstream region, $\rho_{\mathrm{p}, 0}$.

\section{Simulation results}

We first show the phase space plot at time $t=2000 \Omega_{\mathrm{cp}}{ }^{-1}$ in Fig. 1 . The color shows the phase space density in logarithmic scale. The collisionless shock front is located at about $x=57600 \mathrm{c} / \omega_{\mathrm{pp}}$ and the left side region is the upstream region. The shock velocity is $v_{\mathrm{sh}} \approx 40 v_{\mathrm{A}}=1333 \mathrm{~km} / \mathrm{s}$ in 


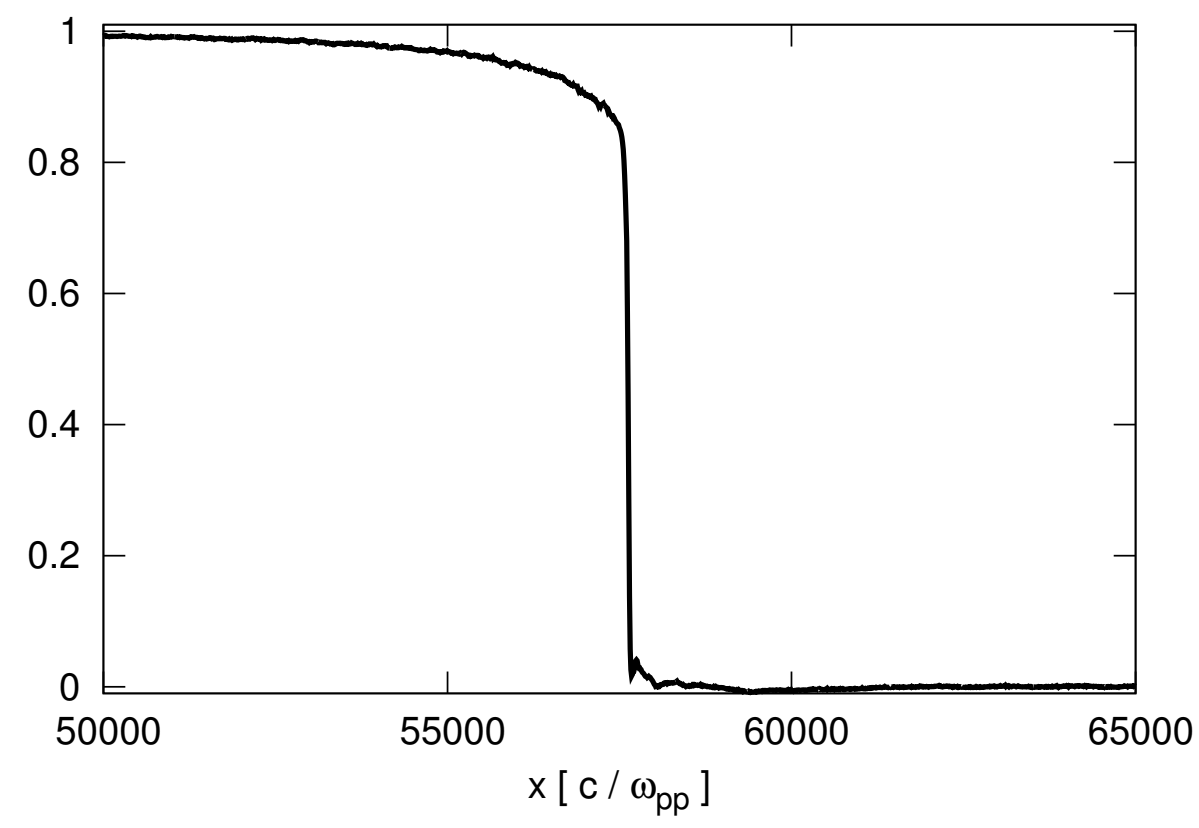

Figure 2: Shock structures averaged over the $y$ direction at $t=2000 \Omega_{\mathrm{cp}}^{-1}$. The black line shows the fluid velocity normalized by the far upstream value, $u_{\mathrm{x}} / v_{\mathrm{d}}$.

the upstream rest frame. By using another Alvén velocity, $v_{\mathrm{A}, \text { tot }}=B_{0} / \sqrt{4 \pi\left(\rho_{\mathrm{p}, 0}+\rho_{\mathrm{H}, 0}\right)}$, defined by the total density, the shock velocity is expressed as $v_{\mathrm{sh}} \approx 57 v_{\mathrm{A} \text {,tot }}$ in the upstream rest frame, where $\rho_{\mathrm{H}, 0}$ is the upstream hydrogen mass density. Many hydrogen atoms leak into the upstream region from the downstream region because they do not interact with electromagnetic fields. The leaking hydrogen atoms originate from hot hydrogen atoms produced by charge exchange between downstream hot protons and downstream hydrogen atoms. Because the collisional ionization dominates over the charge exchange for $v_{\text {rel }}>3000 \mathrm{~km} / \mathrm{s}$, the maximum velocity of leaking neutral particles is expected to be about $3000 \mathrm{~km} / \mathrm{s}$ that is $90 v_{\mathrm{A}}$ in this simulation, which is consistent with our simulation results. The leaking hydrogen atoms are ionized and picked up by the upstream flow and become hot pickup ions. All the upstream cold protons are mainly thermalized at the collisionless shock at $x=57600 \mathrm{c} / \omega_{\mathrm{pp}}$.

Fig. 2 shows $y$-averaged shock structures at time $t=2000 \Omega_{\mathrm{cp}}{ }^{-1}$. The line shows the mean proton velocity of the $x$ direction. As with our previous simulation, in the upstream and downstream regions, the plasma flow is gradually decelerated by the pressure of pickup ions produced in the upstream and downstream regions. However, the shock modification is larger than that of the previous simulation for $v_{\mathrm{d}}=2000 \mathrm{~km} / \mathrm{s}$. This is because more hydrogen atoms leak into the upstream region. According to Ohira (2012) [13], the flux of leaking neutral particles in the shock rest frame, $F_{\text {leak }}$, is given by

$$
\frac{F_{\text {leak }}}{\left(n_{\mathrm{n}}+n_{\mathrm{p}}\right) u_{\mathrm{sh}}}=\frac{1}{36} \frac{\left(1-f_{\mathrm{i}}\right)^{2}}{f_{\mathrm{i}}} \frac{\sigma_{\mathrm{ce}}}{\sigma_{\mathrm{i}}+\sigma_{\mathrm{ce}}} H(r)
$$

where $\sigma_{\mathrm{ce}}$ and $\sigma_{\mathrm{i}}$ are the cross sections of charge exchange and collisional ionization, respectively. 

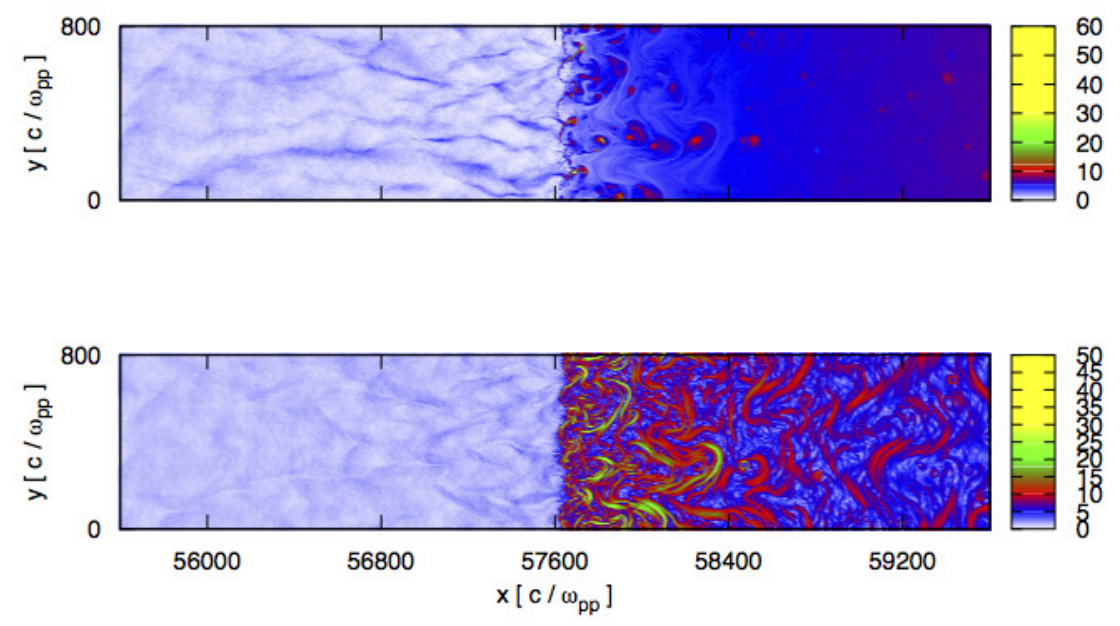

Figure 3: Two-dimensional shock structures at $t=2000 \Omega_{\mathrm{cp}}^{-1}$. The top and bottom panels show the proton density, $\rho_{\mathrm{p}} / \rho_{\mathrm{p}, 0}$ and magnetic field strength, $|B| / B_{0}$, respectively. The left regions $\left(x<57600 c / \omega_{\mathrm{pp}}\right)$ are upstream regions.

$H(r)=6\left(1-2 r^{-1}\right)^{3} /\left(1-r^{-1}\right)$ is a function of the shock compression ratio, $r$, and $H=1$ for $r=4$. $\sigma_{\mathrm{ce}} /\left(\sigma_{\mathrm{ce}}+\sigma_{\mathrm{i}}\right)$ is a decreasing function of the shock velocity, so that the leaking fraction decreases with shock velocity. For this simulation, a few percent of the upstream flux is leaking as the leaking neutral particles, which is consistent with above estimation. We note that the above equation is valid for $f_{\mathrm{i}} \gtrsim 0.5$.

In Fig. 3, we show shock structures of the proton density, $\rho_{\mathrm{p}} / \rho_{\mathrm{p}, 0}$, and magnetic field strength, $|B| / B_{0}$, at time $t=2000 \Omega_{\mathrm{cp}}{ }^{-1}$. Large amplitude density fluctuations are observed both in the upstream and downstream regions. In our previous simulation for $v_{\mathrm{d}}=10 v_{A}=2000 \mathrm{~km} / \mathrm{s} \mathrm{[12]}$, the proton density is correlated with the magnetic field strength in the upstream region. However, in this simulation for $v_{\mathrm{d}}=30 v_{A}=1000 \mathrm{~km} / \mathrm{s}$, unticorrelation is observed in high density filaments of the upstream region although the correlation is observed in many upstream regions. The upstream filamentary structures are produced by compression in the $y$ direction, that is driven by large amplitude Alfvén waves. There is the pressure anisotropy of pickup ions produced by ionization of leaking neutral particles, so that the Alfvén waves are excited by the ion cyclotron instability [8, 12]. The amplitude of the magnetic field fluctuations is $\delta B / B_{0} \approx 3$ in the upstream region. The coherent length scale of magnetic field structures is of the order of the gyroradius of upstream pickup ions.

Fig. 3 shows that the upstream large density fluctuations produce rippled shock structures and turbulence in the downstream region. Then, downstream magnetic fields are amplified by the turbulence. The downstream magnetic fields are locally amplified over $30 B_{0}$ and the spatially averaged value is about $15 B_{0}$ at $x=58400 \mathrm{c} / \omega_{\mathrm{pp}}$. A significant fraction of the upstream kinetic energy is converted to the magnetic field energy. Therefore, we would naively expect that magnetic fields are amplified more strongly for higher Alfvén Mach number shocks.

In our previous simulation for $v_{\mathrm{d}}=10 v_{A}=2000 \mathrm{~km} / \mathrm{s}$ [13], we observed clear mirror mode structures in the downstream region. The mirror mode is excited by the pressure anisotropy of 
pickup ions produced in the downstream region [8]. However, there are no clear mirror mode structures in this simulation because magnetic field lines are not ordered in the downstream region and pickup ions produced in the downstream regions do not have a pressure anisotropy.

\section{Discussion}

The pickup ions are preferentially accelerated by charge exchange and pickup processes [13, 12]. Therefore, the magnetic turbulence observed in this simulation is important for injection into DSA. Simulations in this study are performed in the two-dimensional system, so that particle diffusion perpendicular to the magnetic field line is artificially surpassed [21, 22]. Therefore, we can expect DSA in perpendicular shocks propagating into partially ionized plasmas for a real three dimensional system.

The ratio of the fluid velocity just front of the shock to that just behind the shock is about 3.2 and the total compression ratio is about 4 in this simulation (see Fig. 2). Therefore, if particles are accelerated by DSA in this velocity structure, the momentum spectrum, $d N / d p$, becomes steeper than $p^{-2}[13,14]$.

Our simulation results strongly suggest that particles can be accelerated by collsionless shocks in partially ionized plasmas, which is consistent with observations of Tycho, SN1006, RCW 86, and so on. However, we have not understood whether there is a lower bound of the ionization fraction for DSA or not. This will be addressed in future works.

\section{Acknowledgments}

We thank R. Yamazaki, for useful comments. We also thank ISSI (Bern) for support of the team "Physics of the injection of particle acceleration at astrophysical, heliospheric, and laboratory collisionless shocks". Numerical computations were carried out on Cray XC30 at Center for Computational Astrophysics, National Astronomical Observatory of Japan.

\section{References}

[1] Y. Ohira, K. Murase, and R. Yamazaki, Gamma-Rays from Molecular Clouds Illuminated by Cosmic Rays Escaping from Interacting Supernova Remnants, MNRAS, 410, 1577-1582, 2011

[2] A. R. Bell, The Acceleration of Cosmic Rays in the Shock Fronts-I, MNRAS, 182, 147-156, 1978

[3] R. D. Blandford, and J. P. Ostriker, Particle Acceleration by Astrophysical Shocks, ApJ, 221, L29-L32, 1978

[4] J. Vink, and J. M. Laming, On the Magnetic Fields and Particle Acceleration in Cassiopeia A, ApJ, $584,758-769,2003$

[5] E. G. Berezhko, L. T. Ksenofontov, and H. J. Völk, Confirmation of Strong Magnetic Field Amplification and Nuclear Cosmic Ray Acceleration in SN 1006, A\&A, 412, L11-L14, 2003

[6] A. Bamba, R. Yamazaki, T. Yoshida, T. Terasawa, and K. Koyama, A Spatial and Spectral Study of Nonthermal Filaments in Historical Supernova Remnants: Observational Results with Chandra, ApJ, 621, 793-802, 2005 
[7] Y. Uchiyama, F. A. Aharonian, T. Tanaka, T. Takahashi, and Y. Maeda, Extremely Fast Acceleration of Cosmic Rays in A Supernova Remnant, Nature, 449, 576-578, 2007

[8] J. C. Raymond, P. A. Isenberg J. M. Laming, Non-Maxwellian Proton Velocity Distributions in Nonradiative Shocks, ApJ, 682, 408-415, 2008

[9] Y. Ohira, T. Terasawa, and F. Takahara, Plasma Instabilities as a Result of Charge Exchange in the Downstream Region of Supernova Remnant Shocks, ApJ, 703, L59-L62, 2009

[10] Y. Ohira, and F. Takahara, Effects of Neutral Particles on Modified Shocks at Supernova Remnants, ApJ, 721, L43-L47, 2010

[11] Y. Ohira, Acoustic Instability in the Neutral Precursor Region of Collisionless Shocks Propagating into Partially Ionized Plasmas, MNRAS, 440, 514-518, 2014

[12] Y. Ohira, Simulations of Collisionless Perpendicular Shocks in Partially Ionized Plasmas, PRL, 111, 245002, 2013

[13] Y. Ohira, Effects of Leakage Neutral Particles on Shocks, ApJ, 758, 97(9pp), 2012

[14] P. Blasi, G. Morlino, R. Bandiera, E. Amato, and D. Caprioli, Collisionless Shocks in A Partially Ionized Medium. I. Neutral Return Flux and Its Effects on Acceleration of Test Particles, ApJ, 755, 121(12pp), 2012

[15] J. Giacalone, and J. R. Jokipii, Magnetic Field Amplification by Shocks in Turbulent Fluids, ApJ, 663, L41-L44, 2007

[16] T. Inoue, R. Yamazaki, and S. Inutsuka, Turbulence and Magnetic Field Amplification in Supernova Remnants: Interactions Between a Strong Shock Wave and Multiphase Interstellar Medium, ApJ, 695, 825-833, 2009

[17] H. L. Pauls, G. P. Zank, and L. L. Williams, Interaction of the Solar Wind with the Local Interstellar Medium, J. Geophys. Res., 100, 21,595-21,694, 1995

[18] Y. Ohira and F. Takahara, Absence of Electron Surfing Acceleration in a Two-Dimensional Simulation, ApJ, 661, L171-L174, 2007.

[19] Y. Ohira, and F. Takahara, Oblique Ion Two-Stream Instability in the Foot Region of a Collisionless Shock, ApJ, 668, 320-326, 2008

[20] C. E. Rakowski, M. J. Laming, and P. Ghavamian, The Heating of Thermal Electrons in Fast Collisionless Shocks: The Integral Role of Cosmic Rays, ApJ, 664, 348-357, 2008

[21] J. R. Jokipii, J. Kóta, and J. Giacalone, Prependicular Transport in 1- and 2-Dimensional Shock Simulations, Geophys. Res. Lett., 20, 1759-1761, 1993

[22] J. Giacalone, and J. R. Jokipii, Charged-Particle Motion in Multidimensional Magnetic-Field Turbulence, ApJ, 430, L137-L140, 1994 